\title{
Fondation Sciences Mathématiques de Paris (FSMP)
}

\author{
Gaël Octavia (FSMP, Paris, France)
}

\section{Paris - a fantastic place for doing mathematics}

Paris represents the largest concentration of mathematicians in the world, with a scientific spectrum that is unequalled anywhere else: whatever question you ask in any field of mathematical sciences, there is an expert in Paris able to work on it! Paris also hosts nearly a third of the students enrolled on a mathematics Master's in France.

\section{History of the FSMP}

In 2006, a call for projects was launched by the French government in order to create Thematic Networks for Advanced Research, to promote French research and give it more international visibility and attractiveness. Parisian mathematicians and computer scientists saw this as an opportunity to provide an official framework and adequate administrative structure for an outstanding scientific community and the largest centre of mathematical sciences in the world! The Fondation Sciences Mathématiques de Paris was finally born on 21 December 2006 (https://www.sciencesmaths-paris.fr/).

Over the past few years, the FSMP has consolidated its sources of funding to ensure its sustainability. It has

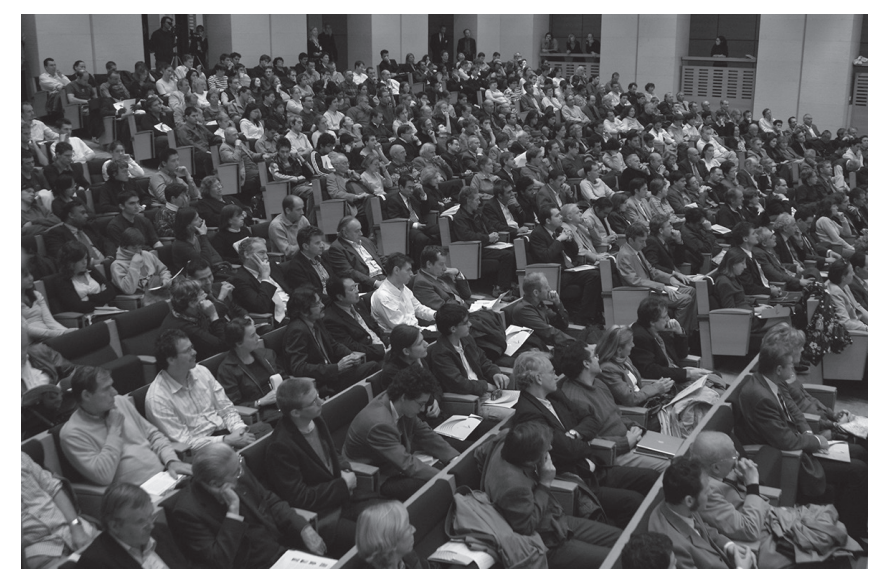

The FSMP's launch day at the Collège de France.

(c) UPMC, Pierre Kitmacher carried forward several projects with success and has received financial support from the French Government, the Région Ile-de-France (the Paris area), the City of Paris and, since 2017, the European Horizon 2020 programme, along with the Marie Sklodowska-Curie COFUND project MathInParis. Furthermore, the FSMP raises funds and receives patronage from individuals and companies.

\section{The FSMP's network}

The FSMP was founded by University Pierre-et-MarieCurie, University Paris-Diderot, CNRS and Ecole Normale Supérieure. It included, from its inception, the four chairs of mathematics of the College de France. In the years that followed its creation, other scientific partners joined and strengthened the network: Inria and the Universities of Paris-Dauphine, Paris-Descartes, Paris 1 Panthéon-Sorbonne, Paris 13 and Paris Sciences et Lettres (teams from the Ecole des Mines, the Observatoire de Paris and EHESS).

The FSMP presently federates 13 laboratories in mathematical sciences and 23 Inria teams. It gathers together more than 1,800 researchers (amongst them 900 permanent posts), including four Fields Medallists, 20 members of the French Academy of Science and many recipients of national and international awards. It is a world leader in mathematical research. Although the Shanghai ranking should be considered with caution, let us recall, in mathematics, that the first university outside the USA is University Pierre-et-Marie-Curie (ranked 3rd) and that among the top 100 are also Ecole Normale Supérieure and the Universities of Paris-Dauphine, Paris-Diderot and Paris 13.

\section{Federating the mathematical community in the Paris area and in France}

The FSMP has succeeded in bringing together all the players in mathematical and theoretical computing research in Paris and its northern suburbs. Following its 
example, similar unifying structures have been founded in the southern suburbs (Fondation Mathématique Jacques Hadamard), the east (Fédération de Recherche Bézout) and the west (Paris-Seine). These structures are scientific partners of the FSMP. Together with Institut Henri Poincaré as well, they organise scientific events and carry out joint projects. This efficient and highly structured network, which the FSMP initiated and heavily contributed to develop, is a great asset in the fight against fragmentation, helping maintain the scientific unity of the mathematical community in the Paris area.

The FSMP also sets up actions to help in federating the French community of mathematicians, such as developing a reference web portal to post all postdoctoral job offers in France and make them more visible and, with other institutions, commissioning a report showing the impact of mathematics on the French economy and society.

\section{The FSMP's programmes}

The FSMP runs and finances four flagship programmes dedicated to outstanding researchers, promising young mathematicians and brilliant students from all over the world:

- The Paris Graduate School of Mathematical sciences (PGSM), which consists of one- or two-year scholarships for Master's students. This programme was pioneered in France. It was immediately a huge success with an average of 300 applications per year, imposing a need for very strong selection. In the framework of the PGSM, the FSMP has set up special programmes in partnership with embassies in order to promote exchanges with their countries: thus, PGSM-Chile, PGSMCuba, PGSM-Iran and PGSM-Romania have emerged, allowing students from these countries to come and follow their Master's degrees in Paris. The programme presently welcomes about 20 students every year.

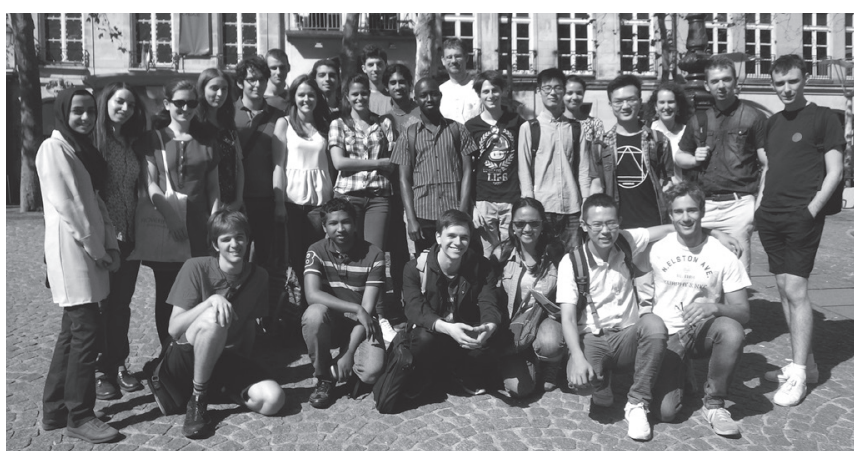

PGSM welcome day. @ FSMP

- The doctoral programme, which provides doctoral allowances and academic and administrative support to $\mathrm{PhD}$ students. This programme has 30 laureates each year.

- The postdoctoral programme, which offers funding and hosting to around 20 talented young researchers every year, for one- or two-year positions. In France, it was the first programme of its kind, conceived in accordance with international selection standards. It has been very successful since 2007, with an average of 150 applications each year. Selection is extremely hard. With this programme, the FSMP competes with the best AngloSaxon universities.

- Excellence Chairs, which offer scientific stays of four to twelve months in laboratories of Paris for top-rank senior researchers or junior researchers with an exceptional profile, in order to create lasting collaborations. The programme welcomes one to three laureates each year, who give courses, organise seminars and participate in many scientific activities during their stay. The laureates receive an attractive salary and additional support to finance students, invite researchers and organise workshops. Researchers among the most renowned in their fields, such as Edward Frenkel, Ron DeVore, Hélène Esnault and the Fields Medallist Timothy Gowers, have been laureates of this programme.

These four main programmes cover all levels of research and training in mathematics, from Master's degree to high-level research activities with international visibility.

The FSMP also finances smaller programmes such as invitations of foreign researchers to its laboratories, funding of scientific stays abroad for $\mathrm{PhD}$ students and the Math $\mathrm{C} 2+$ programme, which consists of giving a taste of mathematics to high school students through lectures, laboratory visits, workshops and courses focused on research approaches.

\section{Integration of PhD laureates}

The FSMP is very concerned with the professional integration of young doctors in mathematics, the $\mathrm{PhD}$ degree still being less valued by companies in France when compared to Anglo-Saxon countries. The FSMP has set up several actions to help them learn more about the job market and the opportunities offered to them, not just in the academic world but also in the economic and industrial worlds, and help them optimise their job searching: e.g. how to write a good resume and how to be effective during a job interview workshops, conferences and meetings with potential employers.

\section{Courses and conferences}

The FSMP participates in the organisation of many courses, conferences and workshops involving members of its laboratories or laureates of its programmes. Its commitment and support relate to the scientific aspects as well as the logistics and communications aspects.

Among the annual events organised by the FSMP, "Mathématiques en mouvement" ("Mathematics in motion") is a conference where students (bachelor's, preparatory classes and Master's) are shown the prodigious diversity of research in mathematics through accessible lectures, round tables, posters exhibitions and meetings with young mathematicians - sometimes $\mathrm{PhD}$ students.

All lectures are filmed and are available on the FSMP's website.

\section{Collaboration with the industrial world}

Strengthening collaboration between academic mathematicians and the industrial world is one of the FSMP's core missions. The FSMP addresses this in a number of ways. 
It promotes relationships between academics and business researchers, co-finances industrial theses, offers continuous training in mathematics and computer science and helps set up doctoral-consulting contracts with companies.

Every year, the FSMP organises with a new industrial partner (EADS, Areva, Huawei...) the conference "Horizon Maths", which brings together academic mathematicians and $R \& D$ researchers in companies on a theme chosen according to the scientific interest of the partner.

The FSMP encourages incubation of start-ups by its young graduates in mathematics (Master's or PhD). It supports Challenge Data, a machine learning competition that offers to students the chance to solve problems of data classification, regression and prediction proposed by start-ups and companies, with real data (medical data, images, sounds, marketing data, internet searches, etc.), giving rise to meetings and creating opportunities.

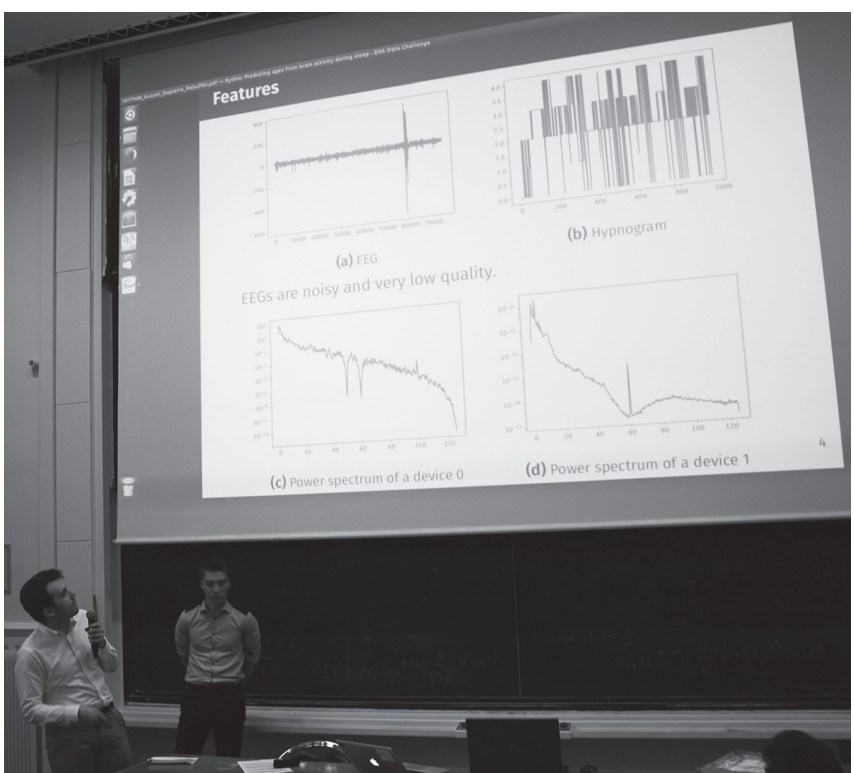

Students solving industrial problems at Challenge Data. @ FSMP

\section{Actions for the general public}

Disseminating mathematical culture outside the community of specialists, and particularly to the general public, is also a mission of the FSMP. This is addressed through various types of media, including short popular articles, video interviews of researchers published on its

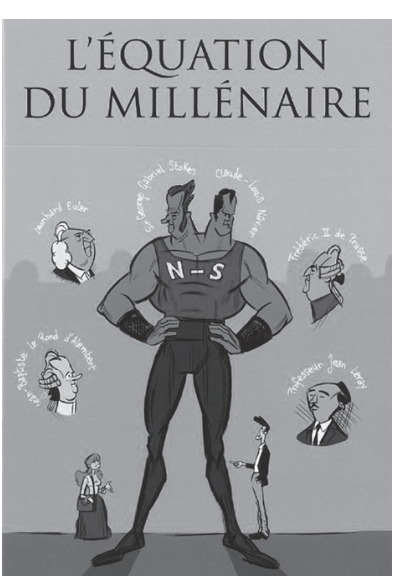
website, blogs and a mathematical calendar every year.

The FSMP is co-editor of Mathématiques, l'explosion continue, a brochure that presents 25 subjects of applied mathematics in 25 accessible articles. It also created and published L'Equation du millénaire (The Millennium Equation), a comic strip about the Navier-Stokes equations.
The FSMP also participates in and supports the holding of flagship events of scientific dissemination, such as the "Salon de la Culture et des Jeux Mathématiques", where 25,000 visitors, including many children and teenagers, come each year to discover mathematics in a playful way.

The FSMP takes part each year in the "Fête de la Science", organising the animation "Raconte-moi ta thèse!" ("Tell me about your thesis!"), a speed meeting between Parisian PhD students and the general public.

It supported financially and contributed to the dissemination of the exhibitions Regards in spaces in dimension 3 and Espace Imaginaires, motifs and mirages designed by Pierre Berger (University Paris-13), a researcher who offers an artistic approach to geometry.

The FSMP also supports the biennial Parity Day, which examines the presence of women in mathematics.

In 2010, none of the major French media sent journalists to Hyderabad to cover the ICM, during which the French mathematicians Cédric Villani and Ngo Bau Chau received the Fields Medal (unlike other nations and even though France was assured of getting at least one Fields Medal). To compensate for this absence, the FSMP produced a blog (which was, at that point, unique), relating the congress day-by-day and often taken up by French journalists left at home, letting the French public know more about the ICM. Four years later, the idea had caught on and several other bloggers shared their experiences of the Seoul Congress (ICM 2014, at which the French-Brazilian mathematician Artur Avila received a Fields Medal).

\section{A look to the future}

Over the last few years, the FSMP has become a key player in mathematical research and training in Paris, in France and internationally. Its significant contribution to the vitality, excellence and attractiveness of the laboratories in its area will continue into the future by drawing outstanding foreign researchers and brilliant foreign students into France and by strengthening the links between research in the mathematical sciences and the world of economics. There are still challenges ahead but the FSMP is willing and able to take advantage of all opportunities to make research in mathematical sciences more successful and contribute to developing and maintaining excellence in its network.

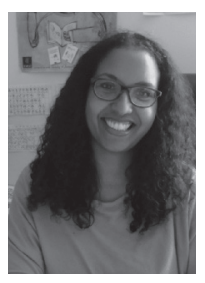

Graduated from Télécom Sud-Paris in 2001, Gaël Octavia was firstly an information systems engineer and then became a scientific journalist. From 2002 to 2008 she works as sub-editor for Tangente, a magazine of mathematical content for the public in general. In February 2008, she joins the FSMP as Communication Manager. She is also a playwright and novelist. 